INPLASY

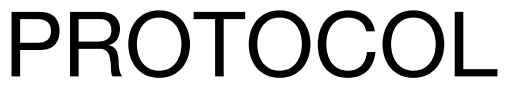

To cite: Xue et al. The efficacy and safety of moxibustion for chronic fatigue syndrome: a systematic review and metaanalysis protocol. Inplasy protocol 202140063. doi: 10.37766/inplasy2021.4.0063

Received: 11 April 2021

Published: 11 April 2021

Corresponding author: Kaiyang Xue

2317649912@qq.com

Author Affiliation:

Guizhou University of

Traditional Chinese Medicine

Support: No: 3040-040200104.

Review Stage at time of this submission: The review has not yet started.

Conflicts of interest:

None declared.

\section{The efficacy and safety of moxibustion for chronic fatigue syndrome: a systematic review and meta-analysis protocol}

Xue, K' Wang, Y²; Wang, X³ Chen, P4; Xiao, C5; Fu, J6; Cui, J7.

Review question / Objective: Is moxibustion effective and safe in the treatment of chronic fatigue syndrome?

Condition being studied: Chronic fatigue syndrome (CFS). Information sources: Literature databases: PubMed, EMBASE, Cochrane library, Sinomed, CNKI, VIP and Wanfang Database; Trial registry: Clinicaltrials.gov, and Chinese Clinical Trial Registry. Source of grey literature: reference lists of relevant reviews.

INPLASY registration number: This protocol was registered with the International Platform of Registered Systematic Review and Meta-Analysis Protocols (INPLASY) on 11 April 2021 and was last updated on 11 April 2021 (registration number INPLASY202140063).

\section{INTRODUCTION}

Review question / Objective: Is moxibustion effective and safe in the treatment of chronic fatigue syndrome?

Rationale: Chronic fatigue significantly reduces the quality of patients' life and even may induce dysfunction, which has become one of the most severe public health problems in the world. Modern medicine mainly supports the treatment of CFS such as anti-anxiety, immune regulation, and nutritional supplementation, but the effectiveness is still controversia. Moxibustion does not invade the body, nor does it need to bear the toxic and side effects of drug metabolism. It is one of the most popular treatment methods for patients. Studies have shown that moxibustion can effectively relieve fatigue, and improve sleep quality, pain and 
paresthesia, gastrointestinal dysfunction and other CFS-related symptoms. Therefore, the purpose of this study is to explore the efficacy and safety of moxibustion for CFS by pooling the current randomized controlled trials, in order to provide high-quality clinical evidence.

Condition being studied: Chronic fatigue syndrome (CFS).

\section{METHODS}

Search strategy: We will systematically search 7 major databases (PubMed, EMBASE, Cochrane library, Sinomed, CNKI (China National Knowledge Infrastructure), VIP (Chinese Scientific Journals Database)and Database Wanfang) and collect the RCTs of moxibustion treatment of CFS. The time range of the search will be from the database to March 31, 2021. We will use a combination of medical subject words and free words to search for terms such as "Chronic Fatigue syndrome", " chronic Fatigue ", "and Moxibustion", a search strategy in PubMed was listed in table1. For including more studies, we will also search the two trial registration platforms ClinicalTrials.gov and Chinese Clinic Trials.gov, as well as references from related reviews.

Participant or population: Patients with CFS, without restrictions on gender, age, course of disease, severity and source of cases.

Intervention: Moxibustion that ignites moxa and produces moxa smoke and temperature is the intervention measure concerned in this study.

Comparator: 1. The experimental group uses moxibustion alone to treat CFS, and the control group uses other methods except moxibustion to treat CFS; 2 . The experimental group uses moxibustion combined with other methods to treat CFS, and the control group uses the same combined method alone as the experimental group to treat CFS.
Study designs to be included: Randomized controlled trials.

Eligibility criteria: We will include patients according to the criteria of the US CDC definition (1988 or 1994 ) for CFS.

Information sources: Literature databases: PubMed, EMBASE, Cochrane library, Sinomed, CNKI, VIP and Wanfang Database; Trial registry: Clinicaltrials.gov, and Chinese Clinical Trial Registry. Source of grey literature: reference lists of relevant reviews.

Main outcome(s): Fatigue Assessment Instrument (FAI).

Additional outcome(s): Fatigue Scale -14 (FS-14), Fatigue Severity Scale (FSS), Pittsburgh Sleep Quality Index (PSQI), natural killer (NK) Cells, interleukin 2 (IL-2), T lymphocyte subsets ((CD4+ , CD8+), cure rate, total efficiency and adverse reactions.

Data management: Two reviewers will independently and repeatedly screen the literatures and extract relevant information, and will cross-check the results. Disagreements will be determined by a third reviewer. The reviewers will first exclude irrelevant literatures by reading the titles and abstracts, then read the full texts to determine the final included RCTs. We will use the standardized tables to extract data from included RCTs. It consists of 3 parts group to: 1) Publishing features: title, first author, establishments, journals, and publication date; 2) patients and treatment: sex, age, source of the patients, sample size, with or without blinding, moxibustion site, moxibustion strength, duration of treatment, adverse reactions, adverse events, follow-up time, loss rate and reasons; 3) outcome data: baseline and follow-up measurement of each index.

Quality assessment / Risk of bias analysis: Two reviewers will independently and repeatedly assess the risk of bias in the included studies and cross-check the results. The disagreement will be determined by a third reviewer. The risk of bias will be assessed using the risk 
assessment tool for randomized controlled trial recommended in the Cochrane handbook 5.1.0. It includes 7 items: 1) random list generation method; 2) allocation concealment; 3) blinding of patients and clinicians; 4) blinding of outcome evaluator; 5) data completeness; 6) selective reporting; 7) other bias sources. Each item will be rated as low, high, or uncertain risk of bias.

Strategy of data synthesis: 1) Meta-analysis for continuous outcomes: weighted mean difference or standardized mean difference and $95 \%$ confidence intervals (Cls) will be used as the effect measures and the inverse variance method will be used to combine the data; 2) Meta-analysis for dichotomous outcomes: relative risks and $95 \%$ Cls will use as the effect measures and the data will be combined using the Mantel-Haenszel method. Cochran's $Q$ test and 12 statistics will be used to evaluate the heterogeneity among studies in the metaanalysis.

Subgroup analysis: Based on the experience of moxibustion therapy in clinical application, when there is obvious heterogeneity in the analysis results, we will analyze the source of the heterogeneity from the following subgroups: 1) Subgroup analysis by moxibustion type: The randomized controlled trial of indirect moxibustion was compared with that of direct moxibustion. It is expected that the former has a better curative effect. 2) Subgroup analysis by treatment course: We will compare randomized controlled trials with a course of treatment $>2$ weeks and $\leq$ 2 weeks. The former is expected to have a better effect.

Sensitivity analysis: In order to verify the robustness of the meta- analysis results, we will conduct the following two sets of sensitivity analyses: 1) excluding studies with high risk of bias; 2) using a fixedeffects model to collect the data from the meta- analysis.

Language: No restrictions.

Country(ies) involved: China.
Keywords: moxibustion, chronic fatigue syndrome, protocol, systematic review, meta-analysis.

Contributions of each author:

Author 1 - Kaiyang Xue.

Email: 2317649912@qq.com

Author 2 - Yanping Wang.

Email: wangyp1119@outlook.com

Author 3 - Xianzhu Wang.

Author 4 - Pingnan Chen.

Author 5 - Caihong Xiao.

Author 6 - Jing Fu.

Author 7 - Jin Cui. 